\title{
Composição Química do Soro de Pintos de Corte Alimentados com Dietas Purificadas ${ }^{1}$
}

\section{Fernanda Alvares da Silva ${ }^{2}$, George Henrique Kling de Moraes $^{2}$, Ana Cláudia Peres Rodrigues ${ }^{3}$, Cláudio César Fonseca ${ }^{4}$, Maria Goreti de Almeida Oliveira ${ }^{2}$, Luiz Fernando Teixeira Albino ${ }^{5}$, Horacio Santiago Rostagno ${ }^{5}$, Heloísa Torres de Freitas ${ }^{5}$}

RESUMO - Um experimento foi conduzido com o objetivo de estudar os efeitos de níveis de ácido L-glutâmico (L-Glu) e vitamina $\mathrm{D}_{3}$ (VD) no soro de pintos de corte de um dia, machos, Hubbard, criados em baterias aquecidas, recebendo dieta básica purificada, suplementada com 5, 10 e $15 \%$ de L-Glu combinados com $0,5.000,10.000$ e 15.000 UI de vitamina $\mathrm{D}_{3} / \mathrm{kg}$. Os animais foram distribuídos em esquema fatorial em um delineamento inteiramente casualizado, com 12 tratamentos, quatro repetições e sete aves por repetição. As concentrações máximas de cálcio no soro $(9,61 \mathrm{mg} / \mathrm{dL})$ foram obtidas com 15\% de L-Glu e 14.181UI de VD; 7,33 mg/dL de cálcio iônico com $15 \%$ de L-Glu e 15.664 UI de VD; 7,20 mg/dL de fósforo com 10\% de L-Glu e 10.863UI de VD. Os maiores valores de atividade de fosfatase alcalina no soro foram obtidos com $10 \%$ de L-Glu e na deficiência de VD. Em geral, as aves alimentadas com $10 \%$ de L-Glu e 15.000UI de VD apresentaram melhor desempenho, melhor perfil sangüíneo e menor incidência de problemas de pernas.

Palavras-chave: ácido L-glutâmico, composição química, pintos de corte, problemas de pernas, soro, vitamina $\mathrm{D}_{3}$

\section{Blood Chemical Composition of Broiler Chicks Fed with Purified Diets}

\begin{abstract}
An experiment was conducted with day-old male Hubbard, broiler chicks, reared in electrically heated batteries, and raised in wire mesh floors and fed purified amino acid diets and water ad libitum for a 14 days period. The purified diets containing all essential amino acids, vitamins and minerals at adequate levels and was supplemented with 5, 10 and 15\% of L-Glu and with $0,5,000$, 10,000 and 15,000 IU of vitamin $\mathrm{D}_{3}$. The experimental design was a factorial $3 \mathrm{x} 4$ with four replicates with seven chicks each. The blood calcium increased up $(9.61 \mathrm{mg} / \mathrm{dL})$ to an estimated level of $15 \%$ of L-Glu and 14,181IU of VD, $7.33 \mathrm{mg} / \mathrm{dL}$ of ionized calcium was obtained with $15 \%$ of L-Glu and 15.664 of VD. The highest blood phosphorus $(7.20 \mathrm{mg} / \mathrm{dL})$ was obtained at an estimated level of $10 \%$ of L-Glu and 10,863IU of VD. The highest blood alkaline phosphatase was obtained at level of $10 \%$ of L-Glu and vitamin D deficiency. Birds fed $10 \%$ L-Glu and 15,000IU VD had better performance, good development of bones, and a lower incidence of leg problems.
\end{abstract}

Key Words: blood, broiler chicks, chemical composition, L-glutamic acid, leg problems, vitamin $\mathrm{D}_{3}$

\section{Introdução}

A rápida taxa de crescimento das aves vem sendo acompanhada por inúmeros problemas, como aumento da deposição de gordura corporal e alta incidência de doenças do sistema esquelético (Sullivan, 1994).

O tecido ósseo é formado por uma estrutura orgânica composta de fibrilas, principalmente colágeno e proteínas não-colagenosas, na qual se deposita a fase mineral, composta principalmente de hidroxiapatita.

Sabe-se que a presença de quantidades adequadas de vitamina D é requerida para o crescimento normal, a maturação e mineralização do osso e para a manutenção do tecido ósseo maduro (Norman \& Hurwitz, 1993; Anderson \& Toverud, 1994).
Rodrigues etal. (1996) observaram que pintos alimentados com 12,5\% de L-Glu apresentaram melhor crescimento ósseo em comparação aos que receberam $5 \%$, confirmando que o nível de nitrogênio não-específico de dietas purificadas é fundamental para o desenvolvimento de pintos, conforme observações feitas por Featherston (1976), Maruyama et al. (1976) e Moraes et al. (1987).

Raquitismo experimental em aves é caracterizado pelo retardonocrescimentocorporal,hipocalcemia,hipofosfatemia, atividade elevada de fosfatase alcalina no soro, hiperplasia da paratireóide e severa distorção óssea (Hedstrom et al., 1986).

Os objetivos deste estudo foram avaliar os efeitos do ácido L-glutâmico (L-Glu) e de vitamina $\mathrm{D}_{3}$ na composição química do soro e nos problemas de pernas de pintos de corte de 1 a 14 dias de idade.

\footnotetext{
${ }^{1}$ Parte da tese do primeiro autor, apresentada à Universidade Federal para obtenção do grau de "Doctor Scientiae" em Zootecnia. Projeto financiado pela Fapemig.

${ }^{2}$ Departamento de Bioquímica e Biologia Molecularda UFV, Viçosa, MG.CEP:36571-000.E.mail:fernanda.silva@mma.gov.br; ghkling@mail.ufv.br 3 Departamento de Bioquímica da UFJF, Juiz de Fora, MG.

${ }^{4}$ Departamento de Veterinária da UFV, Viçosa, MG. CEP: 36571-000.

${ }^{5}$ Departamento de Zootecnia da UFV, Viçosa, MG. CEP: 36571-000.
} 


\section{Material e Métodos}

O experimento foi realizado utilizando-se um delineamento inteiramente casualizado, com esquema fatorial $3 \times 4$ (três níveis de ácido glutâmico $\mathrm{x}$ quatro níveis de vitamina $\mathrm{D}_{3}$ ), totalizando 12 tratamentos, no Setor de Avicultura do Departamento de Zootecnia da UFV.

Foram utilizados 336 pintos de corte, machos, Hubbard, com 1 dia de idade com peso médio de 40 gramas, distribuídos aleatoriamente nos tratamentos. Os tratamentos consistiram de dieta básica purificada (Guimarães, 1988) (Tabela 1) contendo todos os aminoácidos essenciais, vitaminas (exceto vitamina $\mathrm{D}_{3}$ ), minerais e suplementada com três níveis de ácido L-glutâmico (5, 10 e 15\%) combinados com quatro níveis de vitamina D3 $(0,5.000,10.000,15.000$ $\mathrm{UI} / \mathrm{kg}$ ). Cada tratamento foi aplicado a quatro repetições de sete aves cada. As aves foram alojadas em baterias aquecidas com pisos de tela elevados e receberam água e dieta ad libitum durante todo o período experimental de 14 dias.

Ao término do período experimental, seis aves de cada tratamento foram selecionadas ao acaso. A coleta de sangue foi feita por punção cardíaca, e o soro obtido, utilizado para as determinações dos teores de cálcio total, cálcio iônico, fósforo e fosfatase alcalina, no Laboratório de Bioquímica Animal do Departamento de Bioquímica e Biologia Molecular da UFV.

Os soros foram diluídos com água deionizada para a determinação das concentrações de cálcio total, cálcio iônico, fosfato inorgânico e atividade de fosfatase alcalina, por intermédio de procedimentos colorimétricos (espectrofotométricos) usando kits "In Vitro Diagnostica".

As concentrações de cálcio foram determinadas pelo método O-cresolftaleína-complexona (CFC). Em pH alcalino o cálcio reage com CFC formando um complexo molecular de cor púrpura, cuja intensidade de cor é proporcional à concentração do cálcio presente na amostra e possui absorção máxima em $570 \mathrm{~nm}$.

O cálcio iônico foi quantificado a partir das determinações das concentrações de cálcio total $(\mathrm{mg} / \mathrm{dL})$, proteína total $(\mathrm{g} / \mathrm{dL})$ e albumina $(\mathrm{g} / \mathrm{dL})$ no soro sangüíneo, utilizando kits "In vitro Diagnóstica".

Utilizando o método do Molibdato de Amônio, as concentrações de fósforo foram determinadas. $\mathrm{O}$ fósforo inorgânico reage como o molibdato de amônio, formando fosfomolibdato de amônio, que é reduzido a azul de molibdênio, cuja intensidade da cor é proporcional à concentração de íons de fósforo presentes na amostra.

A determinação da atividade de fosfatase alcalina no soro foi feita a partir do método de Roy modificado. A timolftaleína é hidrolisada pela fosfatase alcalina do soro, com liberação de timolftaleína, que em meio alcalino apresenta cor azul, cuja intensidade é diretamente proporcional á atividade enzimática.

A análise estatística dos dados obtidos foi feita por regressão. A escolha do melhor modelo baseou-se

Tabela 1 - Composição da dieta básica purificada Table 1 - Composition of the purified diet

\begin{tabular}{lc}
\hline $\begin{array}{l}\text { Ingredientes } \\
\text { Ingredients }\end{array}$ & $\%$ \\
\hline $\begin{array}{l}\text { Aminoácidos essenciais } \\
\text { Essential amino acids }^{1}\end{array}$ & 8,94 \\
Mistura vitamínica $^{2}$ & 4,48 \\
Vitamin premix $^{2}$ & \\
Mistura mineral $^{3}$ & 9,10 \\
Mineral premix $^{3}$ & 1,00 \\
Bicarbonato de sódio & \\
$\begin{array}{l}\text { Sodium bicarbonate } \\
\text { Óleo de soja }\end{array}$ & 15,00 \\
$\begin{array}{l}\text { Soybean oil } \\
\text { Amido q.s.p. }\end{array}$ & 100,00 \\
Starch & \\
\hline
\end{tabular}

${ }^{1}$ L-Arg. HCl: $1,15 \%$ (L-Arg. HCl); L-His. HCl. $\mathrm{H}_{2} \mathrm{O}: 0,41 \%$ (L-His.HCl.H O); L-Lis.HCl: 1,14\% (L-Lis.HCl);L-Tir: 0,45\% (L-Tir); L-Fen: $0,50 \%$ (L-Fen); L-Trp: 0,15\% (L-Trp);L-Met: 0,35\% (L-Met);L-Cis: 0,45\% (L-Cis);L-Tre: 0,65\% (L-Tre); L-Leu: 1,00\% (L-Leu); L-lle: 0,60\% (L-Ile); L-Val: 0,69\% (L-Val); L-Pro: 0,40\% (L-Pro);Gli: $1,00 \%$ (Gly).

2 Quantidade/kg dieta (Amount $/ \mathrm{kg}$ of diet) - Colina $60 \%: 3,3 \mathrm{~g}$ (Choline); Retinil-Palmitato: 5000 UI (Retinyl palmitate); Colecalciferol: variável (Cholecalciferol); D-a-Tocoferil Acetato: 22 UI ( $D$-a-Tocoferil acetate); Menadiona Sódio Bissulfeto: $2 \mathrm{~g}$ (Menadione sodium bisulfite); Inositol: $1 \mathrm{~g}$ (Inositol); Riboflavina: $9 \mathrm{mg}$ (Riboflavin); Tiamina-HCl: $6 \mathrm{mg}$ (Thiamine.HCl); Pantotenato de Cálcio: $20 \mathrm{mg}$ (Calcium panthotenate); Niacina: $50 \mathrm{mg}$ (Niacin); Piridoxina: $8 \mathrm{mg}$ (Pyridoxine); Ácido Fólico: $2 \mathrm{mg}$; (Folic acid); Biotina: 0,3 mg (Biotin); $\mathrm{B}_{12}(0,1 \%): 20 \mathrm{mg}\left(\mathrm{B}_{12} \cdot 0,1 \%\right) ; \mathrm{BHT}: 0,125 \mathrm{mg}(\mathrm{BHT})$; Excipiente: $30 \mathrm{~g}$ (Excipient) (ROCHE).

${ }^{3}$ Quantidade $/ \mathrm{kg}$ dieta (Amount $/ \mathrm{kg}$ of diet) $-\mathrm{CaCO}_{3}: 18,652 \mathrm{~g}$; $\mathrm{CaHPO}_{4} \cdot 2 \mathrm{H}_{2} \mathrm{O}: 30,53 \mathrm{~g} ; \mathrm{K}_{2} \mathrm{HPO}_{4}: 11,2 \mathrm{~g} ; \mathrm{NaCl}: 6 \mathrm{~g} ; \mathrm{FeSO}_{4}: 0,2 \mathrm{~g}$; ZnO: $0,1225 \mathrm{~g}$; CuSO $\cdot \mathrm{H}_{2} \mathrm{O}: 0,015 \mathrm{~g} ; \mathrm{MnSO}_{4} \cdot \mathrm{H}_{2} \mathrm{O}: 0,51 \mathrm{~g} ; \mathrm{KI}:$ $0,04 \mathrm{~g} ; \mathrm{MgCO}_{3}: 2,5 \mathrm{~g} ; \mathrm{NaMoO}_{4} \cdot 2 \mathrm{H}_{2} \mathrm{O}: 1 \mathrm{~g} ; \mathrm{NaSeO}_{3}: 0,22 \mathrm{~g} ; \mathrm{Casca}$ de arroz: $30,3 \mathrm{~g}$.

EM (ME) (kcal/kg): 2.804

PB (CP) \%: 12,6\% (5\% L-Glu); 17,1\% (10\% L-Glu)e $21,7 \%$ (15\% L-Glu).

Relação Ca: $\mathrm{P}(\mathrm{Ca}: \mathrm{P}$ ratio $)=1,99$. 
no coeficiente de determinação $\left(\mathrm{R}^{2}\right)$ e na significância dos coeficientes de regressão pelo teste $t$ de Student.

\section{Resultados e Discussão}

As concentrações médias de cálcio total, cálcio iônico, fósforo e fosfatase alcalina no soro sangüíneo encontram-se na Tabela 2. O resumo da análise de variância para as concentrações de cálcio total, cálcio iônico fósforo e fosfatase alcalina no soro sangüíneo encontra-se na Tabela 3, na qual podem ser observados efeitos significativos dos tratamentos para as variáveis analisadas.

Em geral, observou-se aumento nas concentrações de cálcio total, cálcio iônico e fósforo no soro sangüíneo com a suplementação de níveis de L-Glu e vitamina $\mathrm{D}_{3}$.

Na Figura 1 observam-se aumentos lineares nas concentrações de cálcio até o valor máximo estimado de $9,61 \mathrm{mg} / \mathrm{dL}$, obtido com $15 \%$ de L-Glu e $14.181 \mathrm{UI}$ de VD.

$\mathrm{Na}$ deficiência de VD, observou-se decréscimo nas concentrações de cálcio sangüíneo total, o que leva a concluir o postulado por Hurwitz (1989), que os sistemas de controle das aves são incapazes de manter a concentração normal de cálcio plasmático na ausência de VD, que resulta no declínio do cálcio plasmático de $10 \mathrm{mg} / 100 \mathrm{~mL}$ para aproximadamente $5 \mathrm{mg} / 100 \mathrm{~mL}$.

Foi necessário um mínimo de 5.000UI de vitamina D para restabelecer as concentrações normais de cálcio no soro, reduzido em função da deficiência de VD.

Foram necessários altos níveis de VD para manter as concentrações de cálcio total no soro no limite fisiológico normal, por meio da intensificação da eficiência de absorção intestinal de cálcio, para manter as funções celulares essenciais e promover a mineralização do esqueleto, e da mobilização dos estoques de cálcio no osso.

A concentração máxima de fósforo no soro (7,20 mg/dL) foi obtida com $10 \%$ de L-Glu e $10.863 \mathrm{UI}$ de VD. O fósforo é um íon abundante em todos os sistemas biológicos, cuja concentração plasmática está mais sujeita a flutuações que o cálcio. Geralmente, a concentração de fósforo é metade do teor

Tabela 2 - Concentrações de cálcio total, cálcio iônico, fósforo e, fosfatase alcalina no soro de pintos de corte Table 2 - Blood total calcium, ionized calcium, phosphorus and alkaline phosphatase levels of broiler chicks

\begin{tabular}{|c|c|c|c|c|c|}
\hline \multirow[t]{2}{*}{$\begin{array}{l}\text { Característica } \\
\text { Trait }\end{array}$} & \multirow[t]{2}{*}{$\begin{array}{l}\text { Vitamina } \mathrm{D}_{3}(\mathrm{UI}) \\
\text { Vitamin } D_{3}\end{array}$} & \multicolumn{3}{|c|}{$\begin{array}{c}\text { Ácido L-glutâmico }(\%) \\
\text { L-glutamic acid }\end{array}$} & \multirow[t]{2}{*}{$\begin{array}{l}\text { Média } \\
\text { Mean }\end{array}$} \\
\hline & & 5 & 10 & 15 & \\
\hline Cálcio total (mg/dL) & 0 & 5,28 & 5,47 & 5,90 & 5,55 \\
\hline \multirow[t]{3}{*}{ Total calcium } & 5.000 & 8,39 & 7,46 & 8,99 & 8,28 \\
\hline & 10.000 & 6,72 & 8,73 & 9,13 & 8,19 \\
\hline & 15.000 & 9,02 & 9,08 & 9,69 & 9,26 \\
\hline Média & & 7,35 & 7,69 & 8,43 & \\
\hline \multicolumn{6}{|l|}{ Mean } \\
\hline Cálcio iônico (mg/dL) & 0 & 4,28 & 4,38 & 4,56 & 4,41 \\
\hline \multirow[t]{3}{*}{ Ionized calcium } & 5.000 & 6,71 & 5,98 & 6,58 & 6,42 \\
\hline & 10.000 & 5,30 & 6,58 & 6,96 & 6,28 \\
\hline & 15.000 & 7,43 & 6,92 & 7,44 & 7,26 \\
\hline Média & & 5,93 & 5,97 & 6,39 & \\
\hline \multicolumn{6}{|l|}{ Mean } \\
\hline Fósforo (mg/dL) & 0 & 3,19 & 4,37 & 6,35 & 4,64 \\
\hline \multirow[t]{3}{*}{ Phosphorus } & 5.000 & 5,18 & 5,39 & 5,06 & 5,21 \\
\hline & 10.000 & 5,85 & 8,08 & 5,27 & 6,40 \\
\hline & 15.000 & 3,74 & 6,45 & 6,13 & 5,44 \\
\hline Média & & 4,49 & 6,07 & 5,70 & \\
\hline \multicolumn{6}{|l|}{ Mean } \\
\hline Fosfatase alcalina (UI/L) & 0 & 235,3 & 262,1 & 259,4 & 252,3 \\
\hline \multirow[t]{3}{*}{ Alkaline phosphatase } & 5.000 & 139,1 & 182,5 & 216,0 & 179,2 \\
\hline & 10.000 & 167,4 & 207,4 & 242,5 & 205,7 \\
\hline & 15.000 & 140,5 & 232,5 & 138,9 & 170,6 \\
\hline Média & & 170,6 & 221,2 & 214,2 & \\
\hline Mean & & & & & \\
\hline
\end{tabular}

R. Bras. Zootec., v.31, n.4, p.1783-1788, 2002 
de cálcio, aproximadamente $5 \mathrm{mg} / \mathrm{dL}$. As mais baixas concentrações de fósforo no soro foram obtidas com $5 \%(3,12 \mathrm{mg} / \mathrm{dL})$ e $15 \%(4,07 \mathrm{mg} / \mathrm{dL})$ e na deficiência de VD.

Corroborando com os resultados encontrados, Perry et al. (1991), Singh \& Grewal (1994) e Goff \& Horst (1995) verificaram decréscimo no cálcio, fósforo plasmático e aumento na atividade de fosfatase alcalina, quando as aves foram alimentadas com uma dieta deficiente em vitamina $\mathrm{D}$ por 14 dias de idade.

Cálcio no soro $=4,6765+0,1075^{* *} \mathrm{~L}-\mathrm{Glu}+0,0004691^{* *}$ VD - $0,00000001654^{* *} V^{2} R^{2}=0,82$

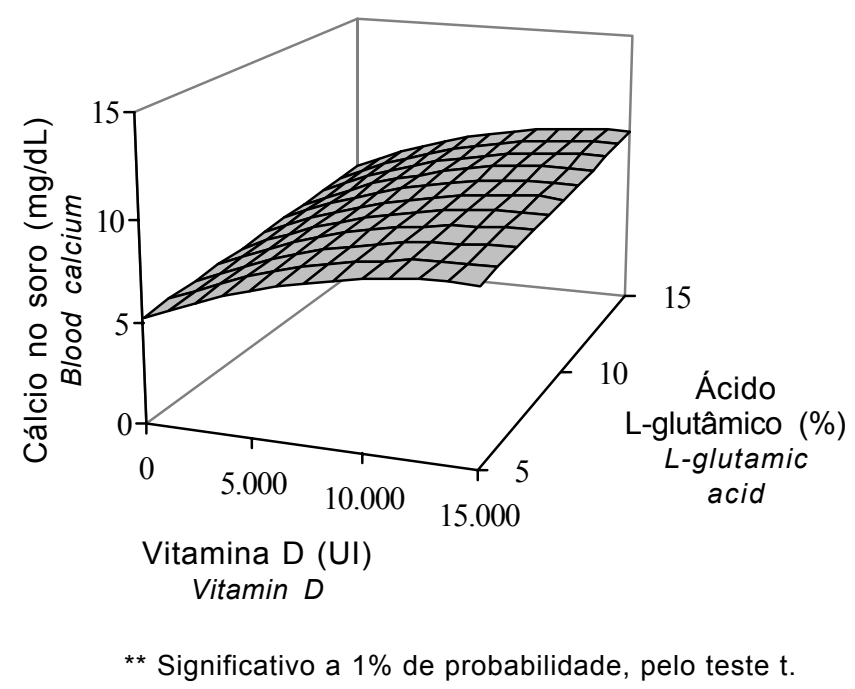

Figura 1 - Estimativa das concentrações de cálcio no soro em função de níveis de vitamina $D$ (VD) e do ácido L-glutâmico (L-Glu).

Figure 1 - Estimate of blood calcium levels in function of vitamin $D(V D)$ and $L$-glutamic acid (L-Glu) levels.
Os resultados sugerem que, aumentando o nível de vitamina $\mathrm{D}$ até uma taxa limite suportável pelo rim para transformá-la em metabólito ativo, aumenta-se a absorção intestinal de cálcio e fósforo, com conseqüente aumento no soro sangüíneo.

Ao contrário do encontrado, Zhouwen \& Tat (1993) não observaram diferença significativa nas concentrações de cálcio, fósforo e fosfatase alcalina no soro sangüíneo das aves que apresentaram problemas de pernas.

Com 5\% de L-Glu as aves apresentaram maior incidência de problemas de pernas (Silva et al., 2001a) e as menores concentrações de cálcio total, cálcio iônico, fósforo e fosfatase alcalina no soro sangüíneo. O mesmo comportamento foi observado por Xu \& Soares Jr. (1998), que observaram pior desempenho das aves, hipocalcemia e raquitismo.

A concentração máxima estimada de cálcio iônico no soro $(7,33 \mathrm{mg} / \mathrm{dL})$ foi obtida com $15 \%$ de L-Glu e 15.664 UI de VD (Figura 2), o que corrobora o obtido por Watkins \& Southern (1991) e Roberson \& Edwards Jr. (1993), os quais observaram aumento significativo no cálcio iônico no soro das aves que receberam maior suplementação de vitamina $D_{3}$.

Foram observados efeitos significativos dos tratamentos nas atividades de fosfatase alcalina no soro de pintos de corte aos 14 dias de idade (Tabela 3).

Os maiores valores de atividade enzimática de fosfatase alcalina foram observados com o nível ótimo de nitrogênio não-específico ( $10 \%$ de L-Glu), coincidentes com as aves que apresentaram melhor desempenho e menor incidência de problemas de pernas (Silva et al., 2001a) e menores concentrações de proteínas não-colagenosas ósseas (Silva et al., 2001b).

Na deficiência de vitamina $\mathrm{D}$, houve aumento na

Tabela 3 - Resumo da análise de variância para as concentrações de cálcio total, cálcio iônico fósforo e fosfatase alcalina no soro

Table 3 - Summary of variance analysis of total calcium, ionized calcium and phosphorus levels

\begin{tabular}{|c|c|c|c|c|}
\hline $\begin{array}{l}\text { Fonte de variação } \\
\text { Source of variation }\end{array}$ & $\begin{array}{l}\text { Cálcio total } \\
\text { Total calcium }\end{array}$ & $\begin{array}{l}\text { Cálcio iônico } \\
\text { Ionized calcium }\end{array}$ & $\begin{array}{c}\text { Fósforo } \\
\text { Phosphorus }\end{array}$ & $\begin{array}{l}\text { Fosfatase alcalina } \\
\text { Alkaline phosphatase }\end{array}$ \\
\hline $\begin{array}{l}\text { L-Glu } \\
(L-G l u)\end{array}$ & $* *$ & Ns & $* *$ & $* *$ \\
\hline $\begin{array}{l}\text { Vitamina } \mathrm{D}_{3} \\
\text { (Vitamin } D_{3}\end{array}$ & $* *$ & $* *$ & $* *$ & $* *$ \\
\hline $\begin{array}{l}\text { L-Glu x vitamina } \mathrm{D}_{3} \\
\left(L-G l u x \text { vitamin } D_{3}\right)\end{array}$ & $*$ & $*$ & $* *$ & $* *$ \\
\hline $\mathrm{CV}(\%)$ & 7,84 & 7,92 & 6,87 & 8,88 \\
\hline
\end{tabular}

* Diferença significativa $(P<0,05)$ (Significant difference $[P<.05])$.

** Diferença significativa $(P<0,01)$ (Significant difference $[P<.01]$ ).

ns Não-significativo $(P>0,05)$ (Not significant $[P>05]$ ).

\section{R. Bras. Zootec., v.31, n.4, p.1783-1788, 2002}


Cálcioiônico $=4,1165+0,04558^{*} L-G l u+0,0003233^{\star \star V D}-0,00000001032^{* \star V D}{ }^{2}$
$R^{2}=0,79$

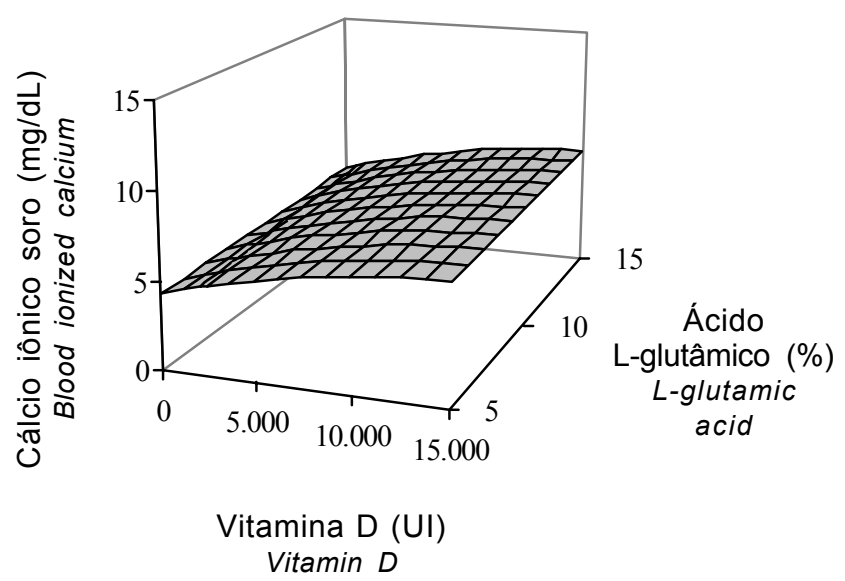

${ }^{* *}$ e * Significativos a $1 \%$ e $5 \%$ de probabilidade, respectivamente, pelo teste $t$.

Figura 2 - Estimativa das concentrações de cálcio iônico no soro, em função de níveis de vitamina $D$ (VD) e ácido L-glutâmico (L-Glu).

Figure 2 - Estimate of blood ionized calcium levels in function of vitamin $D(V D)$ and L-glutamic acid (L-G/u) levels.

atividade de fosfatase alcalina, atribuída ao aumento na atividade dos osteoblastos. Os osteoblastos secretam grande quantidade de fosfatase alcalina, quando estão depositando ativamente a matriz óssea. Acredita-se que essa enzima aumenta a concentração local de fosfato inorgânico ou ative as fibras de colágeno, de modo a causar a deposição de sais de cálcio. Devido à difusão de certa quantidade de fosfatase alcalina no sangue, o nível dessa enzima constitui, em geral, um bom indicador da velocidade de formação óssea (Parthemore et al., 1993; Christenson, 1997).

\section{Conclusões}

As aves que apresentaram maior incidência de problemas de pernas mostraram as mais baixas concentrações de cálcio total, cálcio iônico e fósforo no soro sangüíneo e os maiores valores de atividade enzimática de fosfatase alcalina.

Foi necessário um mínimo de 5.000UI de vitamina $\mathrm{D}_{3}$ para restabelecer as concentrações normais de cálcio no soro, reduzido em função da deficiência de vitamina $\mathrm{D}_{3}$.

\section{Literatura Citada}

ANDERSON, J.J.B.; TOVERUD, S.U. Diet and vitamin D: a review an emphasis on human function. Journal of Nutritional Biochemistry, v.5, n.2, p.58-65, 1994.

CHRISTENSON, R.H. Biochemical markers of bone metabolism: an overview. Clinical Biochemistry, v.30, v.8, p.573-593, 1997.

FEATHERSTON, W.R. Adequacy of glutamic acid synthesis by chick for maximal growth. Poultry Science, v.53, n.6, p.680-686, 1976.

GUIMARÃES, V.M. Efeitos de aminoácidos dispensáveis e indispensáveis no desempenho e anomalias das pernas de pintos de corte. Viçosa, MG: Universidade Federal de Viçosa, 1988. 54p. Dissertação (Mestrado em Agroquímica) - Universidade Federal de Viçosa, 1988.

GOFF, J.P.; HORST, R.L. Assessing adequacy of cholecalciferol supplementation in chicks using plasma cholecalciferol metabolite concentrations as an indicator. Journal of Nutrition, v.125, n.5, p.1351-1357, 1995.

Hedstrom, O.R.; CHEVILle, N.F.; HORST, R.L. Pathology of vitamin D deficiency in growing turkeys. Veterinary Pathology, v.23, n.4, p.485-498, 1986.

HURWITZ, S. Calcium homeostasis in birds. Vitamins and Hormones, v.45, p.173-221, 1989.

MARUYAMA, K.; SUNDE, M.L.; HARPER, A.E. Is L-glutamic acid nutritionally a dispensable aminoacid for the young chick? Poultry Science, v.55, n.1, p.45-60, 1976.

MORAES, G.H.K.; ROGLER, J.C.; FEATHERSTON, W.R. Effects of D-amino acids on growth rate and kidney Damino acid oxidase in chicks. Poultry Science, v.66, n.1, p.98-102, 1987.

NORMAN, A.W.; HURWITZ, S. The role of vitamin D endocrine system in avian bone biology. Journal of Nutrition, v.123, n.2, p.310-316, 1993.

PARTHEMORE, J.G.; BURTON, D.W.; DEFTOS, L.J. Associations and dissociations between serum bone Gla protein and alkaline phosphatase in skeletal metabolism. Journal of Orthopaedic Research, v.11, p.671-676, 1993.

PERRY, R.W.; ROWLAND, G.N.; BRITTON, W.M. Pathology of experimental vitamin D deficiency in turkeys and the effects of various vitamin D supplements. Avian Disease, v.35, p.542-553, 1991.

ROBERSON, K.D.; EDWARDS Jr., H.M. Dietary requirement for 1,25-dihydroxycholecalciferol to prevent tibial dyschondroplasia in broilers. Poultry Science, v.72(Suppl. 1), p.114, 1993.

RODRIGUES, A.C.P.; MORAES, G.H.K.; ROSTAGNO, H.S. Efeitos do ácido L-glutâmico e da vitamina $\mathrm{k}$ no comprimento e na composição química parcial de tíbias e fêmures de pintos de corte. Revista Ceres, v.43, n.249, p.567-580, 1996.

SILVA, F.A.; MORAES, G.H.K., RODRIGUES, A.C.P. et al. Efeitos do ácido L-glutâmico e da vitamina $\mathrm{D}_{3}$ no desempenho e anomalias ósseas de pintos de corte. Revista Brasileira de Zootecnia, v.30, n.6, p.2059-2066, 2001 a. SILVA, F.A.; MORAES, G.H.K.; RODRIGUES, A.C.P. et al. Efeitos do Àcido L-glutâmico e da Vitamina $\mathrm{D}_{3}$ nos fêmures 
e tibiotarsos de pintos de corte. Revista Brasileira de Zootecnia, v.30, n.6, p.2067-2077, 2001 b.

SINGH, A.; GREWAL, G.S. Patho-morphological changes due to rickets in chicks. Indian Journal of Animal Science, v.64, n.11, p.1132-1137, 1994.

SULLIVAN, T.W. Skeletal problems in poultry: estimated annual cost and descriptions. Poultry Science, v.73, n.6, p.879-882, 1994.

XU, T.; SOARES Jr., J.H. Molecular aspects of tibial dyschodroplasia in the chicken: II. Effects of $1,25(\mathrm{OH})_{2} \mathrm{D}_{3}$ on the expression of type $\mathrm{x}$ collagen and alkaline phosphatase activity in growth plate chondrocytes. Nutrition Research, v.18, n.5, p.809-822, 1988.

WATKINS, K.L.; SOUTERN, L.L. Effect of dietary zeolite A and graded levels of calcium on growth, plasma, and tibia characteristics of chickens. Poultry Science, v.70, p.22952303, 1991.

ZHOUWEN, L.; TAT, O.B. Report on cause study of leg weakness in broiler. Acta Veterinaria Sinica, v.24, n.1, p.57-61, 1993.

Recebido em: 14/08/01

Aceito em: 07/05/02 